\title{
Andreev bound states in supercurrent-carrying carbon nanotubes revealed
}

\author{
J-D. Pillet ${ }^{1}$, C. H. L. Quay ${ }^{1 \dagger}$, P. Morfin ${ }^{2}$, C. Bena ${ }^{3,4}$, A. Levy Yeyati ${ }^{5}$ and P. Joyez ${ }^{1 \star}$
}

Carbon nanotubes (CNTs) are not intrinsically superconducting but they can carry a supercurrent when connected to superconducting electrodes ${ }^{1-4}$. This supercurrent is mainly transmitted by discrete entangled electron-hole states confined to the nanotube, called Andreev bound states (ABS). These states are a key concept in mesoscopic superconductivity as they provide a universal description of Josephson-like effects in quantum-coherent nanostructures (for example molecules, nanowires, magnetic or normal metallic layers) connected to superconducting leads ${ }^{5}$. We report here the first tunnelling spectroscopy of individually resolved ABS, in a nanotubesuperconductor device. Analysing the evolution of the ABS spectrum with a gate voltage, we show that the ABS arise from the discrete electronic levels of the molecule and that they reveal detailed information about the energies of these levels, their relative spin orientation and the coupling to the leads. Such measurements hence constitute a powerful new spectroscopic technique capable of elucidating the electronic structure of CNT-based devices, including those with well-coupled leads. This is relevant for conventional applications (for example, superconducting or normal transistors, superconducting quantum interference devices ${ }^{3}$ (SQUIDs)) and quantum information processing (for example, entangled electron pair generation ${ }^{6,7}$, ABS-based qubits ${ }^{8}$ ). Finally, our device is a new type of d.c.measurable SQUID.

First conceived of four decades ago ${ }^{9}$, ABS are electronic analogues of the resonant states in a Fabry-Pérot resonator. The cavity is here a nanostructure and its interfaces with superconducting leads play the role of the mirrors. Furthermore, these 'mirrors' behave similarly to optical phase-conjugate mirrors: because of the superconducting pairing, electrons in the nanostructure with energies below the superconducting gap are reflected as their time-reversed particle-a process known as Andreev reflection. As a result, the resonant standing waves - the ABS - are entangled pairs of timereversed electronic states, which have opposite spins (Fig. 1a); they form a set of discrete levels within the superconducting gap (Fig. 1b) and have fermionic character. Changing the superconducting phase difference $\varphi$ between the leads is analogous to moving the mirrors and changes the energies $E_{\mathrm{n}}(\varphi)$ of the ABS. In response, a populated ABS carries a supercurrent $(2 e / \hbar)\left(\partial E_{\mathrm{n}}(\varphi) / \partial \varphi\right)$ through the device, whereas states in the continuous spectrum (outside the superconducting gap) have negligible or minor contributions in most common cases ${ }^{5}$. Therefore, the finite set of ABS generically determines Josephson-like effects in such systems. As such, ABS a

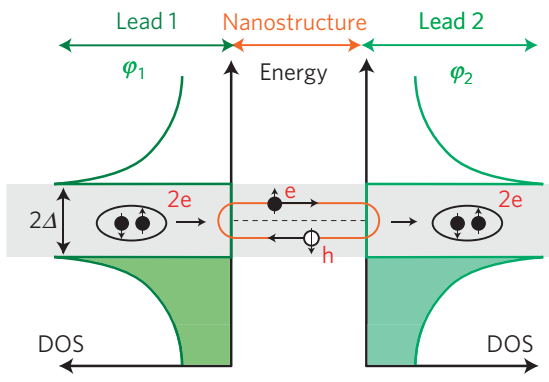

b

c

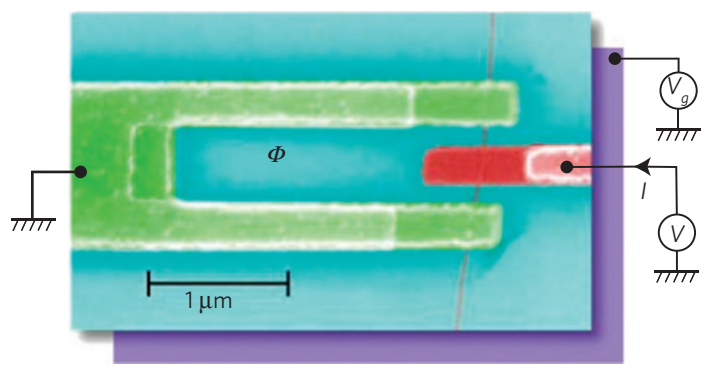

Figure 1 | Principle of ABS and experimental set-up. a, Generic schematic for an $A B S$ in a nanostructure between two superconducting leads, which have DOS with a gap $\Delta$, and with respective superconducting phases $\varphi_{1,2}$. At energies within the superconducting gap (grey band) the Andreev reflection process (which reflects an electron (e) as a hole (h)-its time-reversed particle-and vice versa) leads to the formation of discrete resonant states of entangled e-h pairs confined between the superconductors. These states-the ABS-are electronic analogues to the resonances in an optical Fabry-Pérot cavity. $\mathbf{b}$, The local DOS in the nanostructure is thus expected to exhibit a set of resonances in the gap at the energies of the ABS. The energies of the ABS should depend periodically on the superconducting phase difference $\varphi=\varphi_{1}-\varphi_{2}$, which is analogous to the optical cavity length. c, Colour-enhanced scanning electron micrograph of a device fabricated for the spectroscopy of ABS in a CNT which appears here as the thin vertical grey line. The substrate consists of highly doped silicon serving as a backgate (shown here in violet), with a 1- $\mu$ m-thick surface oxide layer. A grounded superconducting fork (green) is well connected to the tube, forming a loop. The measurement of the differential conductance $\partial I / \partial V$ of a superconducting tunnel probe (red) weakly connected to the tube gives access to the DOS in the CNT, where ABS are confined. The energies of the ABS can be tuned by varying the gate voltage $V_{g}$ and the magnetic flux $\Phi$ threading the loop.

\footnotetext{
${ }^{1}$ Quantronics Group, Service de Physique de l'Etat Condensé, CNRS URA 2426, IRAMIS, CEA, F-91191 Gif-sur-Yvette, France, ${ }^{2}$ Laboratoire Pierre Aigrain (LPA), CNRS UMR 8551, Université Pierre et Marie Curie (Paris VI)—Université Denis Diderot (Paris VII)-Ecole Normale Supérieure de Paris (ENS Paris), France, ${ }^{3}$ Laboratoire de Physique des Solides, CNRS UMR 8502, Université Paris-Sud (Paris 11), Bât. 510, F-91405 Orsay, France, ${ }^{4}$ Institut de Physique Théorique, CEA/Saclay, CNRS URA 2306, Orme des Merisiers, F-91191 Gif-sur-Yvette, France, ${ }^{5}$ Departamento de Física Téorica de la Materia Condensada C-V, Universidad Autónoma de Madrid, E-28049 Madrid, Spain. 'Present address: Laboratoire de Physique des Solides, CNRS UMR 8502, Université Paris-Sud (Paris 11), Bât. 510, F-91405 Orsay, France. *e-mail: philippe.joyez@cea.fr.
} 


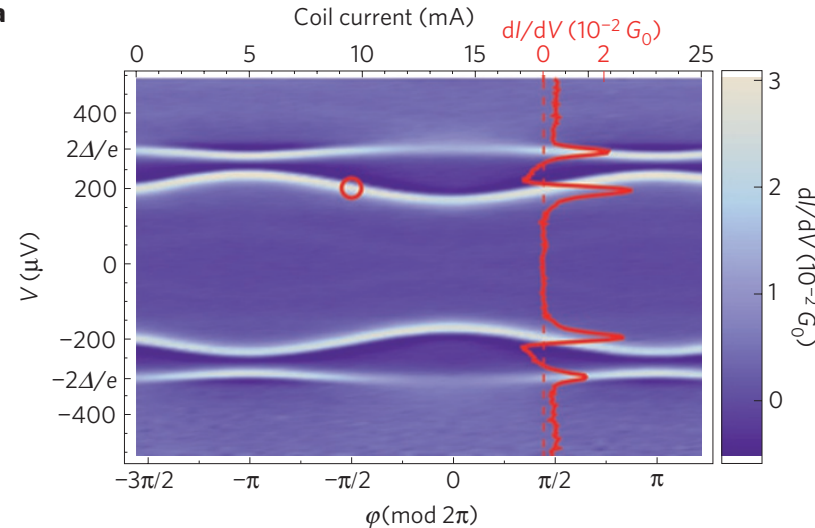

b

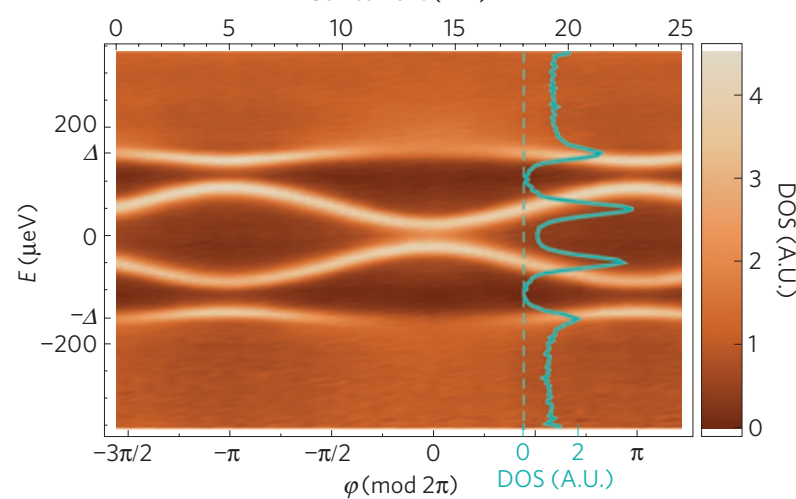

Figure 2 | Flux dependence of the ABS. a, Differential conductance of the tunnel probe at a fixed gate voltage as a function of the bias voltage $V$ of the probe junction (vertical axis) and of the current in a coil (top axis) that controls the flux $\Phi$ through the loop. The sharp resonances are the signature of the $A B S$, and the periodicity of the pattern demonstrates that ABS coherently connect the two end contacts and are sensitive to their superconducting phase difference $\varphi$ (bottom axis). The solid colour traces correspond to cross-sections of the data at the flux indicated by the dashed line. $G_{0}=2 e^{2} / h$ denotes the conductance quantum. $\mathbf{b}$, DOS in the CNT as deconvolved from the data in $\mathbf{a}$, assuming a Bardeen-Cooper-Schrieffer DOS in the tunnel probe. The device can be operated as a d.c.-current SQUID magnetometer by biasing it at a point that maximizes $\partial l / \partial \Phi$, as indicated by a red circle. The fact that the phase is not zero at zero current in the coil is due to a residual magnetic field in our set-up.

play a central role in mesoscopic superconductivity and can be seen as the superconducting counterpart of the Landauer channels for the normal state: in both cases, only a handful of them suffices to account for all of the transport properties of complex many-electron systems such as atomic contacts or CNTs. In effect, the ABS concept quantitatively explains the Josephson effect in atomic contacts ${ }^{10}$; it also explains tunnelling spectroscopy of vortex cores and surface states in some superconductors ${ }^{11}$. However, there has been so far no detailed direct spectroscopic observation of individual ABS. Interest in such spectroscopy has increased with recent proposals for using ABS as quantum bits ${ }^{8}$, and Andreev reflection as a source of entangled spin states ${ }^{6}$.

Nanotubes are particularly good candidates for the observation of ABS. First, CNT-superconductor hybrid systems are expected to show a small number of ABS, and the typical millielectronvolt energy scales involved in nanotube devices are comparable to conventional superconducting gaps. These are favourable conditions for a well-resolved spectroscopy experiment. Second, given the length of CNTs, it is possible to introduce a tunnel probe that enables straightforward tunnelling spectroscopy ${ }^{12}$.
Furthermore, CNTs are of fundamental interest as nearly ideal, tunable one-dimensional systems in which a wealth of phenomena (for example Luttinger-liquid behaviour ${ }^{13}$, Kondo effects ${ }^{3,14}$ and spin-orbit coupling ${ }^{15}$ ) has been observed and the rich interplay of these effects with superconducting coupling has attracted a lot of interest ${ }^{16-22}$.

Our sample is described in Fig. 1. A CNT is well connected to two superconducting metallic contacts $0.7 \mu \mathrm{m}$ apart, leaving enough space to place a weakly coupled tunnel electrode in between. The electrodes are made of aluminium with a few nanometres of titanium as a sticking layer (see Supplementary Information for details); they become superconducting below $\sim 1 \mathrm{~K}$. The two outer contacts are reconnected, forming a loop. A magnetic flux $\Phi$ threaded through the loop produces a superconducting phase difference $\varphi=(2 e / \hbar) \Phi$ across the tube. By measuring the differential conductance of the tunnel contact at low temperature $(T \sim 40 \mathrm{mK}$ ) we observe (see Figs $2 \mathrm{a}$ and $3 \mathrm{a}$ ) well-defined resonances inside the superconducting gap. The energies of these resonances strongly depend on the voltage applied on the backgate of the device, and vary periodically with the phase difference across the CNT, a signature of ABS. From the raw measurement of the differential conductance between the tunnel probe and the loop we can extract the density of states (DOS) in the tube (see for example Fig. 2b) through a straightforward deconvolution procedure (see Supplementary Information). Figure 2 shows the dependence of the ABS spectrum on the flux in the loop at a fixed gate voltage. By d.c.-biasing this device at a point that maximizes $\partial I / \partial \Phi$ (see Fig. 2a), it can be used as a SQUID magnetometer that combines the advantages of refs 23 and 3. Being nanotube-based, our SQUID should be able to detect the reversal of magnetic moments of only a few Bohr magnetons ${ }^{3}$. At the same time, the present device can be read out with a d.c. current measurement (similar to ref. 23) and requires a single gate voltage, making it easier to operate than ref. 3. The gate-voltage dependence of the DOS shows a pattern of resonance lines (Fig. 3b) that is more or less intricate depending on the strength of the coupling to the leads (see Supplementary Information).

We now show that the ABS observed in this device arise from the discrete molecular levels in the CNT. For this we describe our nanotube phenomenologically as a quantum dot coupled to superconducting leads (see Supplementary Information for a detailed discussion of the model). The essential physics of ABS in this system is already captured when one considers a single orbital of the quantum dot filled with either one or two electrons. Owing to the Pauli exclusion principle, these two electrons have opposite spins and can thus be coupled by Andreev reflection. Furthermore, the doubly occupied state is higher in energy by an effective charging energy $\tilde{U}$ that can be determined from the experimental data. Hence, the minimal effective model consists of a spin-split pair of levels (SSPL), the parameters of which are the splitting $\tilde{U}$, the mean position $\bar{E}$ of the SSPL relative to the Fermi level (which is controlled by the gate voltage) and the coupling to the leads (see Supplementary Fig. S1a). Previous theoretical work ${ }^{24,25}$ has shown that there can be up to four ABS, symmetric (in position, but not in intensity) about the Fermi Level. For sufficiently large $\tilde{U}$ (respectively, $\bar{E}$ ), however, the two outer (respectively, all) ABS merge with the continuum and are no longer visible in the spectrum ${ }^{24-26}$.

We now discuss the dependence of the ABS energies on the gate voltage $V_{\mathrm{g}}$. The ABS appear as facing pairs of bell-shaped resonances centred at $\bar{E}\left(V_{\mathrm{g}}\right)=0$ and with their bases resting against opposite edges of the superconducting gap (see the green dashed curves in Fig. 3b.) For large enough $\tilde{U}$ the inner resonances cross at the Fermi energy, forming a loop (Fig. 3b). Such loops are a distinct signature of SSPL in this model (spin-degenerate levels $(\tilde{U}=0)$ cannot give loops). Most of the features observed in Fig. $3 \mathrm{~b}$ can 

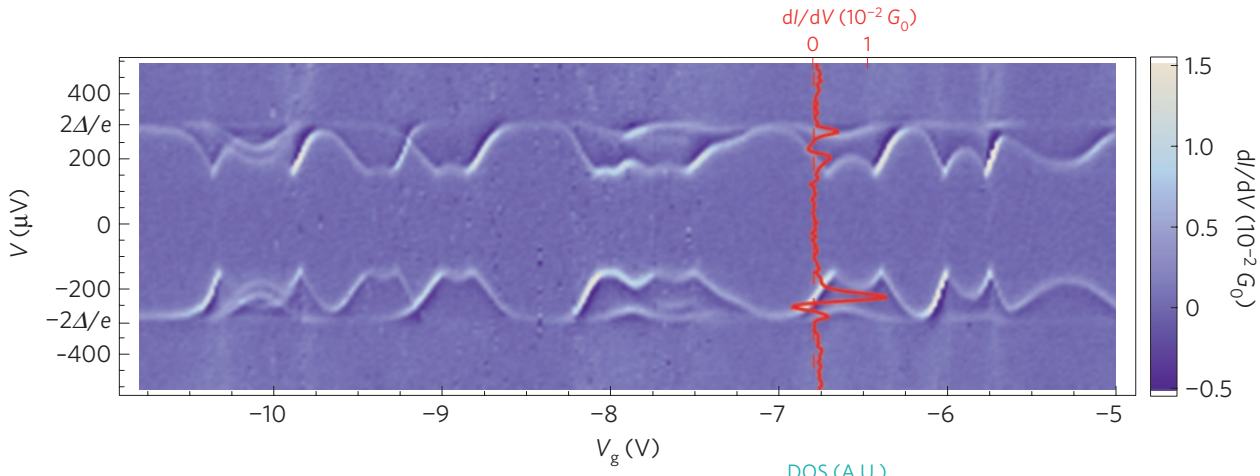

b

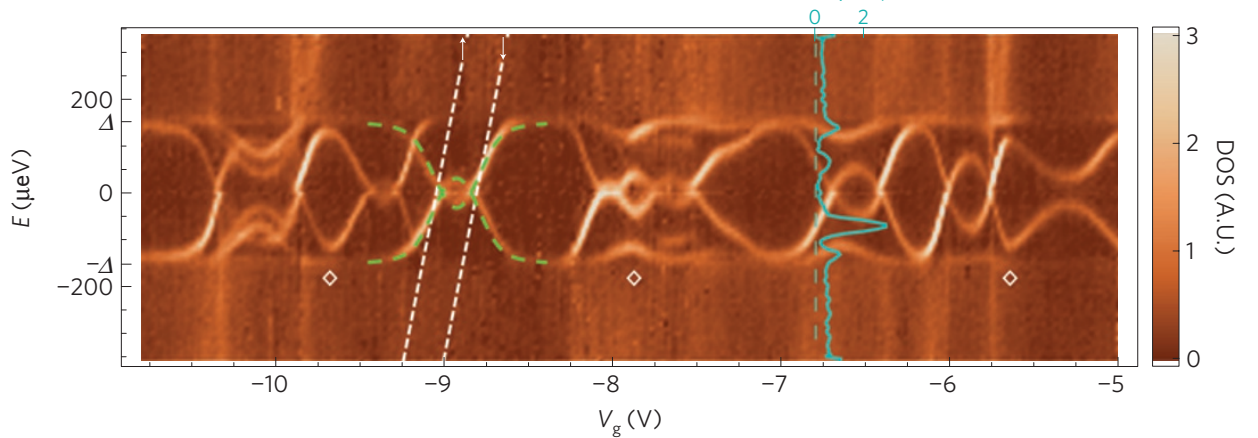

C

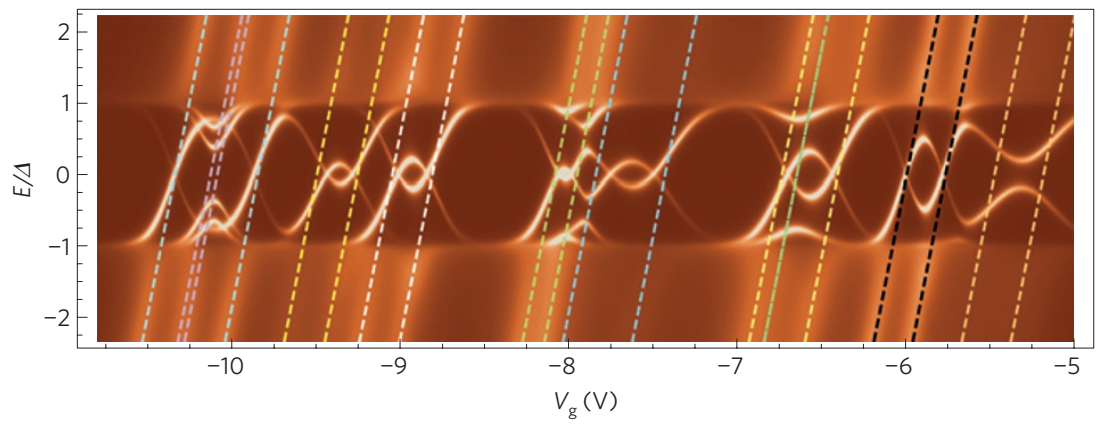

Figure 3 | Gate dependence of the ABS. a, Gate dependence of the differential conductance of the tunnel probe. b, DOS in the CNT as deconvolved from the data in $\mathbf{a}$, after correcting for the gating effect of the probe junction that appears as a slight horizontal shear in $\mathbf{a}$. The ABS form an intricate pattern of intertwined lines. Predicted from a basic quantum-dot model for the CNT, the green dashed bell-shaped lines are the positions of ABS arising from a single SSPL (white dashed lines) crossing the gap as the gate voltage is increased. The spin labelling indicates only the relative orientations of the spins in these levels. Most of the resonances observed in this panel have similar shapes and can be attributed to different SSPL. However, some resonances corresponding to two different SSPL are connected together where indicated by the diamonds. c, Calculated DOS involving several coupled SSPL in a double-quantum-dot model. Here a SSPL is represented by a pair of dashed lines of the same colour. The positions of the levels and their coupling to the electrodes were adjusted to provide best overall agreement with $\mathbf{b}$. This simple model captures many of the observed features and shows how ABS spectroscopy allows the identification of the dot levels, and in particular of their relative spin, without applying any magnetic field.

be identified as such pairs of bell-shaped resonances corresponding thus to different SSPL in the nanotube.

Closer inspection reveals however that adjacent resonances are sometimes coupled, forming avoided crossings (as indicated by diamond symbols in Figs 3b and 4), so that we need to consider the case where two SSPL contribute simultaneously to the spectral properties within the superconducting gap. For this, we extend the model to two serially connected quantum dots each containing a SSPL, with a significant hopping term in between. This model is fairly natural, given that the centre tunnel probe electrode is likely to act as an efficient scatterer. The full description of the model, the derivation of the retarded Green function from which we obtain the spectral properties, and the parameters used to produce the theoretical panels in Figs 3 and 4 are detailed in the Supplementary Information. Assuming for simplicity that all states in the two dots are identically capacitively coupled to the gate and that the couplings to the leads are independent of $V_{\mathrm{g}}$, we can locally reproduce most features of the gate-voltage dependence of the DOS, and simultaneously the flux dependence at fixed $V_{\mathrm{g}}$ (see Fig. 4). By summing contributions of independent SSPL and pairs of coupled SSPL (that is isolated orbitals and coupled pairs of quantum-dot orbitals), we can also reproduce the observed dependence on an extended $V_{\mathrm{g}}$ range (see Fig. 3b,c, and discussion in the Supplementary Information).

Note that a single superconducting terminal is sufficient to induce $\mathrm{ABS}$ in a quantum dot (in which case, of course, there can be no supercurrent, see refs 27,28). Given this, and in light of our analysis, we believe that some features observed in refs 29 , 30 that were tentatively explained as out-of-equilibrium secondorder Andreev reflection can now be reinterpreted as equilibrium ABS spectroscopy on a quantum dot well connected to one superconducting lead, as in refs 27,28 , with the second lead acting as a superconducting tunnel probe.

The agreement between experiment and theory in Figs 3 and 4 shows that $\mathrm{ABS}$ spectra constitute an entirely new spectroscopic tool 

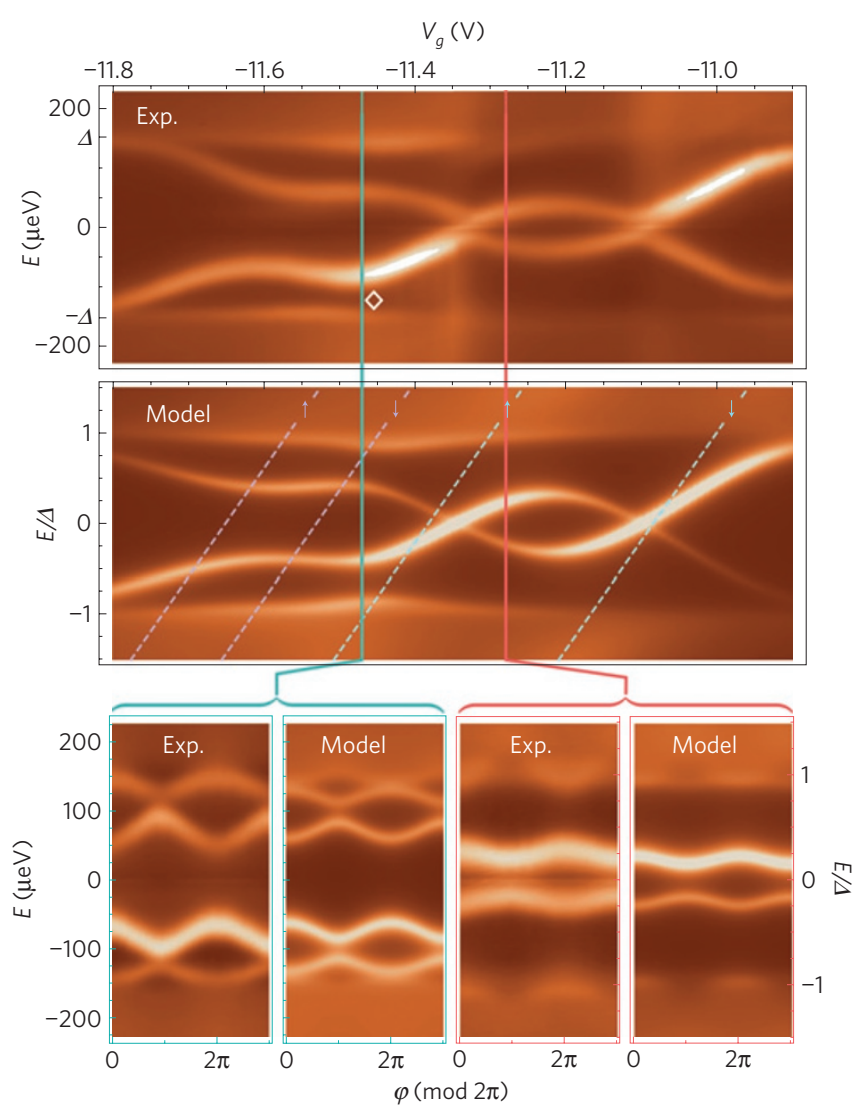

Figure 4 | Details of the gate and phase dependence of the DOS. Top panels: experimental deconvolved DOS as a function of $V_{g}$ and predictions of our double-quantum-dot model, at phase $\varphi=0$. Bottom panels: experimental phase dependence taken at gate voltages indicated by the plain coloured lines in the top panels, and corresponding predictions of the model. All theoretical panels use the same set of parameters; the corresponding level positions are indicated by the dashed lines in the second panel. The spin labelling indicates only the relative orientations of the spins in these coupled levels.

for quantum dots and CNTs. This spectroscopy provides extremely detailed information, in particular about the relative spin state of the nanotube levels, without requiring high magnetic fields. Note that, in contrast to the usual Coulomb blockade spectroscopy of quantum dots, the energy resolution is here essentially independent of the temperature (as long as $k_{\mathrm{B}} T \ll \Delta$ ) and of the strength of the coupling to the leads. It should therefore allow the exploration of the transition between the Fabry-Pérot (where the Luttinger-liquid physics is expected to play a role ${ }^{18,19}$ ) and the Coulomb blockade regimes in CNT. We also expect this new technique to be able to provide key insights in cases where simple charge transport measurements are not sufficient to fully probe the physics at work. In particular, it should allow detailed investigation of the competition between superconductivity and the Kondo effect ${ }^{16}$ that arises for stronger couplings to the leads. Furthermore, used in combination with an in-plane magnetic field, it could also probe spin-orbit interactions ${ }^{20-22}$. Finally, it should be emphasized that although our phenomenological model successfully describes the observed experimental data, further theoretical work is needed to establish a truly microscopic theory that should predict the level splittings from the bare many-body Hamiltonian.

The information extracted from such spectroscopy may also help to optimize field-effect transistors, SQUIDs or even nanoelectromechanical devices based on nanotubes, by better understanding how current is carried through the device. It could also be used for evaluating recently proposed devices for quantum information processing such as entangled electron pair generation by crossed Andreev reflection ${ }^{6}$ or ABS-based quantum bits ${ }^{8}$. Regarding the latter, our observation of tunable ABS is heartening even though the measured spectroscopic linewidth $(30-40 \mu \mathrm{eV}$ full-width at half-maximum) seems to question the feasibility of such qubits (if it were intrinsic to the sample, it would correspond to subnanosecond coherence time). The present linewidth is however likely to be caused simply by spurious noise in the experimental set-up. More investigations are needed to assess the potential of nanotube $\mathrm{ABS}$ as qubits.

Received 30 April 2010; accepted 8 September 2010; published online 14 November 2010; corrected online 17 November 2010

\section{References}

1. Kasumov, A. Y. et al. Supercurrents through single-walled carbon nanotubes Science 284, 1508-1511 (1999).

2. Jarillo-Herrero, P., van Dam, J. A. \& Kouwenhoven, L. P. Quantum supercurrent transistors in carbon nanotubes. Nature 439, 953-956 (2006).

3. Cleuziou, J. P., Wernsdorfer, W., Bouchiat, V., Ondarcuhu, T. \& Monthioux M. Carbon nanotube superconducting quantum interference device. Nature Nanotechnol. 1, 53-59 (2006).

4. Pallecchi, E., Gaass, M., Ryndyk, D. A. \& Strunk, C. Carbon nanotube Josephson junctions with Nb contacts. Appl. Phys. Lett. 93, 072501 (2008).

5. Beenakker, C. W. J. in Transport Phenomena in Mesoscopic Systems (eds Fukuyama, H. \& Ando, T.) (Springer, 1992).

6. Recher, P., Sukhorukov, E. V. \& Loss, D. Andreev tunneling, Coulomb blockade, and resonant transport of nonlocal spin-entangled electrons. Phys. Rev. B 63, 165314 (2001).

7. Herrmann, L. G. et al. Carbon nanotubes as Cooper-pair beam splitters. Phys. Rev. Lett. 104, 026801 (2010).

8. Zazunov, A., Shumeiko, V., Bratus', E., Lantz, J. \& Wendin, G. Andreev level qubit. Phys. Rev. Lett. 90, 087003 (2003).

9. Kulik, I. Macroscopic quantization and proximity effect in S-N-S junctions. Sov. Phys. JETP-USSR 30, 944-947 (1970).

10. Della Rocca, M. L. et al. Measurement of the current-phase relation of superconducting atomic contacts. Phys. Rev. Lett. 99, 127005 (2007).

11. Fischer, Ø., Kugler, M., Maggio-Aprile, I., Berthod, C. \& Renner, C. Scanning tunneling spectroscopy of high-temperature superconductors. Rev. Mod. Phys. 79, 353-419 (2007).

12. Chen, Y., Dirks, T., Al-Zoubi, G., Birge, N. O. \& Mason, N. Nonequilibrium tunneling spectroscopy in carbon nanotubes. Phys. Rev. Lett. 102, 036804 (2009).

13. Bockrath, M. et al. Luttinger-liquid behaviour in carbon nanotubes. Nature 397, 598-601 (1999).

14. Buitelaar, M. R., Nussbaumer, T. \& Schönenberger, C. Quantum dot in the Kondo regime coupled to superconductors. Phys. Rev. Lett. 89, 256801 (2002).

15. Kuemmeth, F., Ilani, S., Ralph, D. C. \& McEuen, P. L. Coupling of spin and orbital motion of electrons in carbon nanotubes. Nature 452, 448-452 (2008).

16. Glazman, L. I. \& Matveev, K. A. Resonant Josephson current through Kondo impurities in a tunnel barrier. JETP Lett. 49, 659-662 (1989).

17. Spivak, B. I. \& Kivelson, S. A. Negative local superfluid densities: The difference between dirty superconductors and dirty Bose liquids. Phys. Rev. B 43, 3740-3743 (1991).

18. Fazio, R., Hekking, F. W. J. \& Odintsov, A. A. Josephson current through a Luttinger liquid. Phys. Rev. Lett. 74, 1843-1846 (1995).

19. Caux, J., Saleur, H. \& Siano, F. Josephson current in Luttinger liquid-superconductor junctions. Phys. Rev. Lett. 88, 106402 (2002).

20. Dell'Anna, L., Zazunov, A., Egger, R. \& Martin, T. Josephson current through a quantum dot with spin-orbit coupling. Phys. Rev. B 75, 085305 (2007).

21. Dolcini, F. \& Dell'Anna, L. Multiple Andreev reflections in a quantum dot coupled to superconducting leads: Effect of spin-orbit coupling. Phys. Rev. B 78, 024518 (2008).

22. Zazunov, A., Egger, R., Jonckheere, T. \& Martin, T. Anomalous Josephson current through a spin-orbit coupled quantum dot. Phys. Rev. Lett. 103, 147004 (2009).

23. Giazotto, F., Peltonen, J. T., Meschke, M. \& Pekola, J. P. Superconducting quantum interference proximity transistor. Nature Phys. 6, 254-259 (2010)

24. Yoshioka, T. \& Ohashi, Y. Numerical renormalization group studies on single impurity Anderson model in superconductivity: A unified treatment of magnetic, nonmagnetic impurities, and resonance scattering. J. Phys. Soc. Jpn 69, 1812-1823 (2000).

25. Vecino, E., Martín-Rodero, A. \& Levy Yeyati, A. Josephson current through a correlated quantum level: Andreev states and pi junction behavior. Phys. Rev. B 68, 035105 (2003). 
26. Meng, T., Florens, S. \& Simon, P. Self-consistent description of Andreev bound states in Josephson quantum dot devices. Phys. Rev. B 79, 224521 (2009).

27. Deacon, R. S. et al. Tunneling spectroscopy of Andreev energy levels in a quantum dot coupled to a superconductor. Phys. Rev. Lett. 104, 076805 (2010).

28. Dirks, T. et al. Andreev bound state spectroscopy in a graphene quantum dot. Preprint at http://arxiv.org/abs/1005.2749 (2010).

29. Eichler, A. et al. Even-odd effect in Andreev transport through a carbon nanotube quantum dot. Phys. Rev. Lett. 99, 126602 (2007).

30. Grove-Rasmussen, K. et al. Superconductivity-enhanced bias spectroscopy in carbon nanotube quantum dots. Phys. Rev. B 79, 134518 (2009).

\section{Acknowledgements}

This work was partially supported by ANR project ANR-07-BLAN-0240 SEMAFAET, C'Nano project SPLONA and Spanish MICINN under contracts NAN2007-29366 and
FIS2008-04209. The authors gratefully acknowledge discussions with the Quantronics group, O. Auslaender, J. C. Cuevas, R. Egger, M. Grifoni, T. Kontos, H. le Sueur, A. Martín-Rodero, P. Simon and C. Strunk.

\section{Author contributions}

J-D.P. and C.Q.H.L. fabricated the sample. J-D.P., C.Q.H.L. and P.J. designed and carried out the experiment. J-D.P., C.Q.H.L., P.J., C.B. and A.L.Y. analysed the data and wrote the paper. P.M. provided the equipment for the nanotube synthesis.

\section{Additional information}

The authors declare no competing financial interests. Supplementary information accompanies this paper on www.nature.com/naturephysics. Reprints and permissions information is available online at http://npg.nature.com/reprintsandpermissions. Correspondence and requests for materials should be addressed to P.J. 